\section{POLARIZATION OF SOUTHERN PULSARS}

Tasman Derk van Ommen

Physics Department, University of Tasmania, Hobart Tasmania 7001 June 1992

Polarimetric data are presented for a total of eighty-two (mostly) southern pulsars, recorded at an observing frequency of $950 \mathrm{MHz}$. Thirty-two of these sources were observed with a high-speed integrator, constructed as part of this project.

The integrator has been designed to digitize up to sixteen receiver channels and compute average pulse profiles in real-time. The instrument is capable of recording singlepulse as well as averaged data and has a maximum sample rate in excess of $800 \mathrm{kHz}$.

For many of the pulsars observed, there is no published polarimetry, and for most, these observations constitute the only data between $630 \mathrm{MHz}$ and $1612 \mathrm{MHz}$. Analysis of the integrated profiles shows properties which are in general agreement with extrapolations from other frequencies. The data are also consistent with the view that orthogonal position-angle modes become more prevalent at high frequency. The majority of pulsars display features which accord with current core-cone geometrical interpretations, although a significant number require polarimetry at other frequencies in order to resolve their classification. Integrated profile mode-changing has been identified for the first time in PSR1358-63. Analysis of the position-angle variation in the interpulse and main-pulse of PSRs 1055-52 and 1702-19 has confirmed high inclinations between magnetic and rotation axes as reported in the literature.

Sequential observations at offset frequencies have permitted computation of rotation measures for seven of the pulsars studied. Five of these values are new results, and one is a refinement of an existing value.

Several single-pulse observations have been made of PSR1451-68, permitting detailed study of the emission from this pulsar. These observations reveal extreme variability in pulse shape and intensity on pulse-to-pulse timescales. Emission statistics suggest a significant fraction of null pulses and fluctuation analyses show no organized modulation. Both polarization properties and component morphology are found to vary with pulse intensity. The polarization properties of this pulsar are unusual, and are not consistent with the rotating vector model. The emission shows prominent quasi-orthogonal position-angle modes across most of the profile. Circular polarization is found to be correlated with position-angle and, at some longitudes, two distinct polarization modes can be identified which are approximately antipodal when projected onto the Poincaré sphere.

\section{NONLINEAR WAVE PROCESSES IN COLLISIONLESS PLASMAS}

\section{Douglas John Percival \\ Department of Theoretical Physics, University of Sydney, NSW 2006} June, 1992

Several contributions to the theory of nonlinear wave processes in nonrelativistic collisionless plasmas are presented in this thesis. The approach adopted is the description of the plasma response to an electromagnetic field disturbance using a hierarchy of linear and nonlinear response ten- sors. The material naturally divides into two parts treating unmagnetised and magnetised plasmas separately.

In Part I, two distinct research interests concerning the response tensors of an unmagnetised plasma are pursued. In Chapter 1, a novel construction of the linear and nonlinear response tensors for an isotropic plasma is presented in 4tensor notation. The construction uses gauge invariance, charge continuity conditions and symmetry properties of the response tensors to constrain the coefficients of an arbitrary linear combination of tensors. Chapter 2 details the calculation of the quadratic response tensor describing all second order plasma processes in a warm unmagnetised plasma, with particle species satisfying a Maxwellian velocity distribution. The quadratic response tensor is expressed in terms of a set of generalised plasma dispersion functions evaluated in Chapter 3. The application of the exact quadratic response tensor to specific three-wave interactions is discussed in Chapter 4.

Part II examines several phase coherent four-wave processes in plasmas subject to an ambient magnetic field. An approximate form of the effective cubic response tensor for a magnetised plasma is derived in Chapter 5 from the general 4-tensor expressions for the quadratic and cubic response tensors. A compact nonlinear dispersion equation describing a wide class of parametric and modulational instabilities involving thermally modified magnetoionic waves is derived in Chapter 6, and used to discuss the effect of the magnetic field on wave stability. In Chapter 7 , the generalisation of the well-known Zakharov equations describing modulational and wave collapse phenomena to include magnetic field effects is examined, and a lagrangian formulation of the magnetised Zakharov equations is presented. In Chapter 8 , phase conjugate reflection by degenerate four-wave mixing in a magnetised plasma is analysed for arbitrary field strengths and interaction geometries. In Chapter 9, the analysis of a recently identified modulational instability driven by counterpropating Langmuir waves and leading to fundamental emission near the plasma frequency is generalised to account for unequal pump wave strengths and the effects of an ambient magnetic field. The application of the instability in models of solar Type III emission is critically discussed.

\section{CHEMICALLY INHOMOGENEOUS SOLAR MODELS AND THE SOLAR NEUTRINO PROBLEMS}

\section{Stuart J Robinson}

Department of Mathematics, Monash University, Clayton Vic 3168 March 1993

A new class of evolutionary solar models which possess a small, metal-rich core are examined and shown to yield a possible solution to the long standing solar neutrino problem. For a core of $\sim 0.025 \mathrm{M}_{\odot}$, it is possible to produce a current age solar model, having the observed values of luminosity radius and surface hydrogen abundance yet yielding a flux of neutrinos, as seen by the Davis, ${ }^{37} \mathrm{Cl}$ neutrino detector, reduced by some $50 \%$ when compared to that predicted by a standard solar model. 